Open Access

\title{
An investigation of cross-linguistic transfer between Chinese and English: a meta-analysis
}

\author{
Man Yang(D, North Cooc and Li Sheng*
}

\footnotetext{
* Correspondence: Isheng@udel.edu Department of Communication Sciences and Disorders, University of Delaware, 540 South College Ave, Newark, DE 19713, USA
}

\begin{abstract}
Cross-linguistic transfer embodies language learners' use of linguistic knowledge of their first language to leverage the learning of a second language. The cross-linguisitc transfer between Chinese and English has been studied by scholars from different disciplines. However, variances and inconsistencies exist among prior studies regarding the investigated linguistic domains and reported results. Therefore, a meta-analysis is needed to systematically investigate the cross-linguistic transfer between Chinese and English. This meta-analysis presents research on cross-linguistic transfer between the two languages in four domains: phonological awareness, decoding skills, vocabulary, and morphological awareness. Using 33 articles conducted in different countries, our results show small to moderate levels of transfer in the above four domains. In addition, it was found that the results were moderated by geographic location of the study and participant age. Overall, the meta-analysis indicates that English and Chinese share common linguistic features that can allow for transfer in learning between the languages. Bilingual learners can benefit in educational environments that tap into these linguistic features.
\end{abstract}

Keywords: Cross-linguistic transfer, Chinese, English

\section{Introduction}

Chinese is one of the most widely spoken languages worldwide. Nearly a quarter of the world's population speaks Chinese as their native language (Hua, 2002). Recent immigration trends indicate a rapid increase of Chinese-speaking population in many parts of the world. For example, a recent U.S. census reported that nearly three million people spoke Chinese at home, making it the third most spoken language in United States after English and Spanish (U.S. Census Bureau, 2013). A large number of children who speak Chinese as their first language (L1) are also learning English as the second language (L2) in the United States, Canada, UK, and China. The linguistic dynamics between the two languages have motivated researchers to examine their relationship because transfer plays an important role in second language acquisition (e.g., Bialystok et al., 2005; Tong \& McBride-Chang, 2010; Wang et al., 2009; Zhang et al., 2012; Yeung and Ganotice, 2014).

There is growing research evidence of cross-linguistic transfer in the process of second or foreign language learning among alphabetic languages. For example, studies

(C) The Author(s). 2017 Open Access This article is distributed under the terms of the Creative Commons Attribution 4.0 International License (http://creativecommons.org/licenses/by/4.0/), which permits unrestricted use, distribution, and reproduction in any medium, provided you give appropriate credit to the original author(s) and the source, provide a link to the Creative Commons license, and indicate if changes were made. 
have revealed that bilingual children's linguistic skills, such as phonological awareness and morphological awareness in their home language, predict their acquisition of English literacy (e.g., Adams, 1990; Casalis \& Louis-Alexandre, 2000; Durgunoğlu, 2002; Riches \& Genesee, 2006; Snow et al., 1998;Wagner \& Torgesen, 1987). In addition, a number of studies examining learning English as an L2 among children who speak an alphabetic L1 like Spanish, French or Italian have found that students' L1 proficiency level can facilitate their L2 learning (e.g., Cisero \& Royer, 1995; Comeau et al., 1999; Durgunoglu et al., 1993; D’Angiulli et al., 2001). Despite the evidence of positive crosslinguistic transfer between alphabetic languages, there are fewer studies investigating cross-linguistic transfer between non-alphabetic and alphabetic languages, such as Chinese and English.

Whether and to what extent cross-linguistic transfer occurs between Chinese and English is a compelling question given the clear differences between the two languages. Chinese is phonologically different from English in terms of the sound inventory, syllable structures, and the use of tones to differentiate the meaning of syllables. Tone is a unique feature of Chinese and Mandarin Chinese tones can be classified into five categories: high level, rising, falling-rising, falling, and neutral (Cheng, 1991). In contrast to English, Chinese syllables do not have consonant blends or clusters (Hashimoto, 1972). English also has more phonemes than Chinese; for instance, English phonemes /v/ or / z/ cannot be found in Chinese. The two languages also differ in terms of morphology. Chinese does not utilize inflectional morphemes, through which grammatical features such as tense and plurality are expressed. In terms of orthography, Chinese has a distinct written form compared to English in that Chinese uses strokes to form characters whereas English uses letters to form words. In terms of vocabulary, Chinese and English do not share any cognates (i.e., words that share visual, phonological, and semantic similarities between languages) orthographically and have only a handful of cognates phonologically.

A comprehensive understanding of cross-linguistic transfer regarding phonological awareness, decoding, vocabulary, and morphological awareness between Chinese and English will enable us to better understand how learning one language impacts the acquisition of a second language when the two languages are historically and typologically unrelated. It will help school teachers and language and reading specialists understand how two distant languages may or may not interact with each other during the process of language acquisition. This will in turn facilitate the design of suitable education and intervention programs for bilingual Chinese-speaking students. Previous studies on cross-linguistic transfer between Chinese and English show large variation both in the specific linguistic domains examined and in results (e.g., Gottardo et al., 2001; Pasquarella et al., 2011; Wang, Cheng \& Chen, 2005; Yeung \& Ganotice, 2014; Zhang \& Koda, 2014). A meta-analysis can provide a comprehensive review of the literature and quantify evidence of linguistic of transfer across multiple studies.

Melby-Lervag and Lervag (2011) conducted a meta-analysis to examine the crosslinguistic transfer of reading related skills between both alphabetic and ideographic L1 s and English as L2. They found correlations between L1 and L2 in terms of oral language, phonological awareness and decoding. Moreover, instructional setting and age were found to have moderator effects on the aforementioned relationships. Specifically, learners who were receiving L1 and L2 instruction at schools showed stronger 
cross-language correlations in the domain of decoding than learners who were receiving L2 only instruction. With regard to age, some of the skills showed increased correlation with increases in learners' age (e.g., the correlation between L2 oral language and L2 reading comprehension) whereas others showed decreased correlations with increases in learners' age (e.g., the correlation between L1 decoding and L2 comprehension). To the best of our knowledge, their study is the only meta-analysis on crosslinguistic transfer. However, their study examined cross-linguistic transfer among various language pairs with the majority of studies examining alphabetic language pairs. Therefore, we do not know if and to what extent their findings of cross-linguistic transfer can be generalized to language pairs that share fewer similarities. Our meta-analysis places an exclusive focus on Chinese and English and included articles published since 2000 to examine the overall strength of cross-linguistic correlations by different linguistic domains. In addition, we aim to detect potential moderators that might influence the magnitude of correlations across studies.

\section{Cross-linguistic transfer}

Cross-linguistic transfer is defined as language learners' use of linguistic knowledge of one of their languages to leverage the learning of another language. Theories on crosslinguistic transfer shed light upon the role of L1 in promoting L2 or foreign language learning. For example, the linguistic interdependence hypothesis by Cummins (1979) suggests that L1 and L2 acquisition is mutually dependent and both contribute to and draw from the same common underlying proficiency. In other words, language minority students' L2 development is dependent upon their L1 proficiency. In the school context, L1 can be maintained as long as the outside environment provides sufficient stimulation, in the meantime, the intensive exposure of L2 in school can contribute to rapid bilingual development without compromising the development of L1 (Verhoeven, 1994). Therefore, the degree to which language transfer differs depends on the children's proficiency level in their L1, as well as the context under which the children are learning the languages; cross-linguistic transfer is more likely to occur if children's L1 continues to develop.

Apart from the interdependence model (Cummins, 1978, 1979), the contrastive analysis theory emphasizes the use of detailed linguistic analysis to identify structural similarities as well as differences between the two languages to make predictions regarding when and how cross-linguistic transfer may take place (e.g., Connor, 1996; Ellis, 1994; Lado, 1957; Odlin, 1989). According to this framework, cross-linguistic structural similarities may accelerate L2 acquisition and structural differences may impede L2 acquisition (Melby-Lervag \& Lervag, 2011). With these theoretical frameworks in the backdrop, in the next section of this paper, we will discuss several linguistic areas of interest.

\section{Linguistic areas of interest}

Previous research findings have well documented that phonological awareness, vocabulary, decoding and morphological awareness are related to and predictive of students' reading development, regardless of the nature of L1 (e.g., Deacon \& Kirby, 2004; Huang and Hanley, 1995; Keenan et al., 2008; Metsala, 1999; Shanahan et al., 2006; Shu et al., 
2006). Therefore, it is important for us to investigate the relationship between Chinese as L1 and English as L2 in terms of the above four areas to further illustrate the language acquisition process among young Chinese speakers who are acquiring English as their second language. In addition, in order to fill up the gap of the review conducted by Melby-Lervag and Lervag in 2011 in which they examined cross-linguistic transfer of phonological awareness, oral language, and decoding, we also included morphological awareness in our review to investigate the relationship between Chinese and English in a more thorough and comprehensive perspective.

\section{L1 Vs. L2 phonological awareness}

Phonological awareness refers to the ability to segment speech into smaller phonological units and analyze and manipulate these units. Phonological awareness has been proven to be an essential skill in early reading acquisition regardless of the type of orthography (Wagner \& Torgesen, 1987). Studies reveal cross-linguistic transfer from Chinese to English phonological awareness primarily at the syllable and phoneme levels (e.g., Cheung et al., 2010; Chien et al., 2008; Chow, 2014; Chow et al., 2005; Chung et al., 2013; Luo et al., 2014; Wang et al., 2005). Research supports the conclusion that Chinese phonological awareness is predictive of English phonological awareness, and such association seems to be bi-directional (e.g., Chien et al., 2008; Chow, 2014).

\section{L1 Vs. L2 vocabulary}

Vocabulary, also known as oral vocabulary, is commonly measured using instruments that assess receptive and expressive vocabulary knowledge and listening comprehension. It is different from visual word recognition, which is often times known as decoding skills. Similar to what Melby-Lervag and Lervag (2011) discussed in their review, we focus on the cross-linguistic transfer of oral vocabulary in this particular area. The Revised Hierarchical Model (RHM, Kroll, \& Stewart, 1994; Kroll et al., 2010) proposes that bilinguals possess a shared conceptual store along with two separate word-form lexicons when learning two languages (Sheng et al., 2012). In the earlier stage of L2 learning, bilinguals typically need their L1 vocabulary as the mediator to access L2 vocabulary due to weak direct links between conceptual representation and L2 lexicon. The use of L1 lexicon as a bridge will fade away as bilinguals gain more L2 proficiency. Hence, the connection between L1 and L2 lexicon serves as a prerequisite for later direct linkage between L2 vocabulary and conceptual representation. The RHM provides an additional theoretical foundation for our hypothesis of the relationship between L1 and L2 vocabulary. According to the RHM, the L1-L2 vocabulary relationship should be moderated by the bilinguals' L2 learning duration and L2 proficiency.

\section{L1 Vs. L2 decoding}

Decoding is a skill measured by accuracy of word identification in English or character identification in Chinese. Logographic languages like Chinese differ vastly from alphabetical languages such as English in the representation of orthographic forms. Chinese can be characterized as a morpho-syllabic writing system whereas English allows units such as syllables and words to be assembled from letter-phoneme mappings (Wang et al., 2003). It has been propsed that reading Chinese may utilize more the direct route from orthographic representations to semantic representations, whereas reading English 
may rely more on the indirect route which converts written symbols to their phonological representations before accessing the semantic meanings (Wang et al., 2003). Even though reading Chinese characters may not involve the learners's phonological knowledge to the same extent as reading English, research studies support the conclusion that there is a clear and important role of phonological awareness in reading Chinese (e.g., Chen, Flores d' Arcais, \& Cheung, 1995; Zhou \& Marslen-Wilson, 1996). Given these commonalities in the decoding of the two orthographies, we therefore hypothesize a significant relationship between Chinese and English decoding skills.

\section{L1 Vs. L2 morphological awareness}

Morphological awareness refers to children's ability to recognize word parts and use these parts to form new words. Previous studies have confirmed the importance of morphological awareness in predicting the development of vocabulary, word reading, and reading comprehension among alphabetic languages (e.g., Carlisle, 2000; Deacon \& Kirby, 2004; Nagy, Berninger, \& Abbott, 2006) as well as in Chinese (e.g., Wang, Cheng, \& Chen, 2006; Zhang \& Koda, 2014). In addition, researchers have identified crosslinguistic transfer between Chinese and English primarily concerning the decomposition and formation of compound words. This is due to similarities in compound word structure in the two languages (e.g., snowman, street light) (Lam \& Sheng, 2016; Luo et al., 2014; Zhang et al., 2010; Zhang et al., 2012). Therefore, we expect to find a correlation between Chinese and English morphological awareness specifically with regard to learner's awareness of compound formation rules.

\section{Current study}

The current study seeks to review studies which reported correlations between L1 (Chinese) and L2 (English) in the domains of phonological awareness, decoding, vocabulary, and morphological awareness. We were unable to find enough studies to conduct meta-analyses on other linguistic domains such as reading comprehension or spelling. We hypothesize that cross-language correlations, if any, would be moderated by two factors: the learning context embedded in three different geographic locations (Hong Kong/Singapore; Mainland China/Taiwan; US/Canada), and grade levels. The three geographic locations covered in the current literature represent natural environmental variation when it comes to the amount of L1 and L2 support available to the learners. At one end, Chinese-speaking bilinguals living in the United States or Canada receive L2 (English) only instruction at school. Chinese (or heritage language) instruction, when available, is provided on an informal basis through the family or the community. These after-school or weekend heritage language programs tend to be very short in duration and insufficient to support the continuous growth of their L1 (Sheng, 2014). At the other end, in Mainland China and Taiwan, Mandarin Chinese is the official language in the greater society and the language of instruction in school. All students are required to learn English as a foreign language at their school but the age of onset and the intensity of English instruction differ. Moreover, exposure to English outside of the classroom can be quite limited in Mainland China and Taiwan. Lastly, in Hong Kong and Singapore, both Chinese and English are official languages and both are widely used and promoted in public places and in the media. In Singapore, English is the first school language and the main medium of instruction in all national schools, 
while Mandarin Chinese is mainly offered as L2 (Pakir, 2004). However, children frequently receive Mandarin exposure through the television and radio. In Hong Kong, although numerous efforts regarding language policy promoting educational interests of students have been made by both pre- and post-handover governments, the choice of medium of instruction was eventually left to individual schools so students may receive various amount of formal support of their L1 and L2 (Tung, Lam, \& Tsang, 1997). Cantonese, English and Mandarin are the three official languages in Hong Kong with Cantonese currently being spoken by more than $95 \%$ of its population (Kan \& Adamson, 2010). Compared to Cantonese, however, English has a higher status in Hong Kong society and is very popular with key stakeholders such as parents, teachers and schools given the stronger association between its competence and social or economic advantages (Choi, 2003; Kan \& Adamson, 2010). Therefore, there are more pedagogical emphases on English learning in Hong Kong society, which provides students more opportunities to get exposed to English and in the meantime learning their first language through school.

Similar to what Melby-Lervag and Lervag (2011) found in their study, we anticipate that cross-linguistic transfer is more likely to take place when both languages are widely supported, either through formal education or informal exposure. With regard to the effect of grade level, based on the Revised Hierarchical Model (Kroll \& Stewart, 1994; Kroll et al., 2010), we expect that in the early phases of L2 acquisition, learners may rely more on their L1 skills hence greater cross-language correlations in lower grade levels.

\section{Review}

Method

Literature search

The systematic search of the articles was situated in electronic database such as ERIC, PsycInfo, Education source, and EBSCO with the descriptors cross-linguistic, transfer, bilingual* (truncation), Chinese L1 learners, English L2 learners, English language learners, English L2, English additional language, Cantonese L1 learners, Mandarin L1 learner, limited English speakin, phon* awareness, morpho* awareness, oral language, reading, decoding, comprehension, and vocabulary. Chinese is an umbrella term for at least seven mutually unintelligible languages and numerous additional regional languages mostly associated with minority groups (Sun, 2006). Here we focus on Mandarin and Cantonese as these two languages are the most widely spoken and most researched Chinese languages. An additional search was conducted in Google scholar and individual journals that are likely to publish relevant articles such as Reading and Writing, Scientific Studies of Reading, International Journal of Bilingualism,Educational Psychology, Asia-Pacific Educational Researcher, and Applied Psycholinguistics. This first phase yielded a total of 37,819 articles.

\section{Inclusion criteria}

All studies were carefully examined by two trained undergraduate students to filter out the ones that did not meet our inclusion criteria. Specifically, we only included studies that were 1) peer-reviewed, 2) written in English, 3) focused on Chinese and English, 4) targeted bilingual language learners who were exposed to English from early grades 
(e.g., kindergarten or before 3rd grade) to high school, 5) reported correlations between Chinese (L1) and English (L2). After applying all the inclusion criteria, a total of thirythree studies conducted in various locations such as Hong Kong, Taiwan, mainland China, Canada, Singapore and United States were finalized for our further analysis with a total of 4564 participants. Articles that were excluded tended to lack reported correlation results, focus on adult learners only, and focus on either Chinese or English but not both. For the purpose of our study, we also excluded intervention studies that measured effects of specific interventions. Figure 1 displays the flowchart of our literature search process.

\section{Coding process}

All studies were coded using a list of variables. This included publication year, sample size, participants' age, geographic location, study design (cross-sectional or longitudinal), grade level, measurements, target constructs (e.g., phonological awareness, vocabulary, decoding skills, morphological awareness), language of instruction, current exposure to Chinese and English, onset time of English learning, and family SES. Only the first time point data was coded for longitudinal studies. For cross-sectional studies, we separately coded the correlation results by grade level or language dominance, if available. When coding outcome variables, we focused on correlations for the target linguistic domains between the two languages and excluded measurements such as nonverbal IQ, and working memory. If studies utilized multiple measures for each target construct, a normalization value was calculated by averaging correlation coefficients. For example, measures that focused on syllable, onset, rime/rhyme, and phoneme were all included for the calculation of an average correlation for phonological awareness per study. Measures that specifically focused on Chinese tone awareness were coded separately from other phonological awareness skills such as phoneme

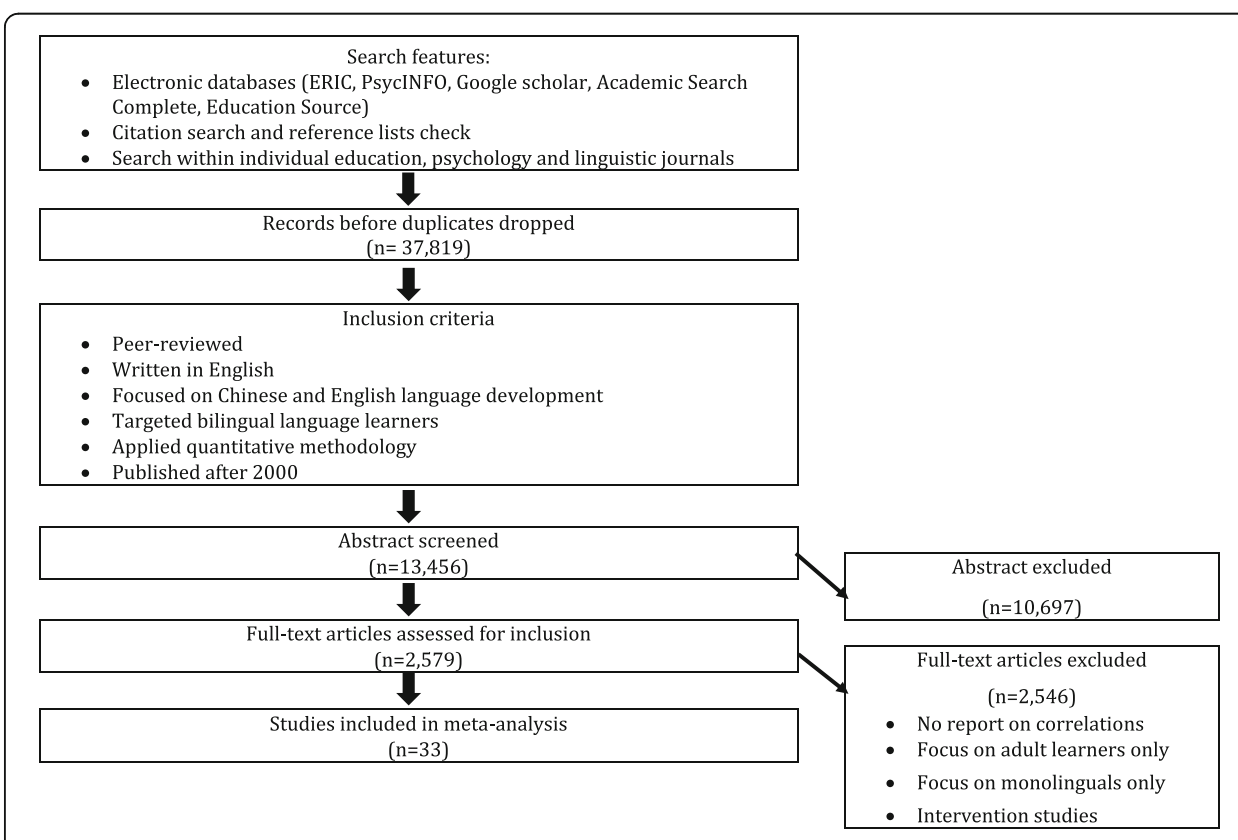

Fig. 1 Flow chart for the literature search and inclusion of studies (adapted from Melby-Lervag \& Lervag, 2011) 
and syllable. Decoding skills were measured through Chinese character reading and English word reading tasks. Vocabulary in English was measured using receptive vocabulary instruments such as the Peabody Picture Vocabulary Test (PPVT, Dunn \& Dunn, 1997) and Chinese vocabulary was primarily measured using receptive assessments such as the Chinese PPVT (Dunn \& Dunn, 1997). Only a small number of studies $(n=2)$ utilized expressive tasks to measure Chinese vocabulary, and so we only used receptive Chinese scores in the following analysis. For the coding of instructional language, we only coded studies in which explicit statement regarding teachers' language of instruction was made. For example, studies were coded if they contained statement like "participants attended local primary schools where Cantonese was the teaching medium" (Chung \& Ho, 2010, p.199). The same rule also applied to current exposure to both languages as well as the onset time of English learning and only studies with explicit statement were coded. We created an Excel spreadsheet to collect and compile all the information mentioned above from each selected study. A random sample of $30 \%$ of the studies were chosen to be coded by two raters to examine interrater reliability so that the accuracy of the coding could be assured. We used Kappa coefficients to adjust for chance agreement between raters and the Kappa coefficient $(\mathrm{K}=.8)$ was considered good across coded study characterstics (Altman, 1990). The coding inconsistencies were further resolved through discussions among coders.

Theme of correlation Each theme has to meet the criterion of having at least eight studies reporting the correlation between L1 and L2. A total of four major themes were identified and explored in this review and they are phonological awareness, decoding skills, vocabulary and morphological awareness between Chinese as L1 and English as L2.

Meta-analysis procedures We hypothesized that the meta-analysis would reveal a range of correlations for target linguistic domains with significant between-study variation. Therefore, a random effect model was more appropriate for the current study (Lipsey \& Wilson, 1993). We used a software program named 'comprehensive metaanalysis' for the majority of our analyses (Borenstein, Hedges, Higgins, \& Rothestein, 2009). The program was chosen because it is one of the most widely used software programs specialized for meta-analysis (Bax et al., 2007). Correlations between L1 and L2 with regard to the target domains such as phonological awareness, decoding, vocabulary, and morphological awareness were entered into predefined data sheet in the program together with the study name and sample size of each study. The effect sizes were displayed using Pearson correlation. A 95\% confidence interval (CI) was also reported for each study to examine whether the overall correlation was statistically different from zero. The overall correlation was calculated using a weighted average of correlations from each of the studies along with a $\mathrm{z}$ statistic to examine whether the overall correlation was different from zero. In cases where studies reported correlation coefficients separately by age, gender, outcome variable, language dominance and geographic locations, the datasets in those studies were further divided into independent subsets for further meta-analysis.

In order to examine whether the variation in correlations between studies was significant, we used a $Q$-test of homogeneity (Hedges \& Olkin, 1985). A significant $Q$ test result indicates variability among correlations in the selected studies. $I^{2}$, the proportion of 
total variation between correlations that is caused by real heterogeneity rather than chance, was further calculated to measure the magnitude of heterogeniety.

To conduct a moderator analysis based on categorical variables, we separated studies based on the groups or levels of the target categorical variable. The $Q$ statistic was separated into within-group and between-group parts to examine whether variation among study subsets was statistically different based on moderators (Kim \& Quinn, 2013; Hasselblad \& Hedges, 1995). A significant between-group $Q$ test result indicates the existence of moderator effect. Besides $Q$ test, we also compared the correlation magnitude with CIs to examine the differences between subsets of studies. Two categorical variables were included for moderator analysis: geographic location and participants' grade level. Rules were set for the minimal number of studies required for each level of the moderator variables. Specifically, we determined that at least three studies were needed for each level of the moderators to qualify for the analysis on this particular moderator (Melby-Lervag \& Lervag, 2011).

\section{Results}

The results are reported according to a total of four themes. Among the numerous linguistic features addressed by the 33 studies, we compiled similar ones into four major categories. For example, we grouped onset, rime, phonological, syllable or phoneme awareness under the theme of phonological awareness; character reading, word/ pseudo-word reading, and reading fluency in L1 and L2 were categorized into the theme of decoding; vocabulary knowledge in L1 and L2 was placed under the theme of vocabulary; and derivational or compound morphological skills were grouped under the theme of morphological awareness. We reported the overrall correlations between L1 and L2 for each of the four themes.

\section{Descriptive characteristics of studies}

Major charateristics for each study in the meta-analysis are summarized using percentages in Table 1. A total of thirty-three articles were included for further analysis. Roughly $61 \%$ of the studies were published between 2010 and 2016, and $46 \%$ of the studies were conducted in Hong Kong, recruited primary grade (36.36\%) participants, and utilized cross-sectional (81.82\%) study design. Besides Hong Kong where most of the studies were conducted (45.45\%), there were similar numbers of studies conducted in the US (15.15\%), Canada (12.12\%), and mainland China (12.12\%), followed by multiple locations (9.09\%), Taiwan (3.03\%) and Singarpore (3.03\%). The majority of the studies (73\%) did not report on family socioeconomic status (SES). Current exposure to both languages was not reported consistently: some studies $(n=5)$ reported the number of hours participants received on a daily basis of each language at school; some $(n=10)$ briefly mentioned the experience of language without providing too much details, and the remaining $(n=18)$ did not mention it at all. In terms of onset of English learning, 6 studies included participants who started to learn English varying from 3 to 6 years old and 10 studies had participants' onset of English learning ranging from kindergarten to third grade.

\section{Correlation between L1 and L2 phonological awareness}

A total of 28 independent correlations comprising 3001 children examined the relationship between L1 and L2 phonological awareness. The age range of the samples varied 
Table 1 Main characteristics of studies included for analysis

\begin{tabular}{|c|c|}
\hline Study characteristics & All studies $(N=33)$ \\
\hline \multicolumn{2}{|l|}{ Publication year } \\
\hline 2000-2009 & 39.39 \\
\hline 2010-now & 60.61 \\
\hline \multicolumn{2}{|l|}{ Study design } \\
\hline Cross-sectional & 81.82 \\
\hline Longitudinal & 18.18 \\
\hline \multicolumn{2}{|l|}{ Study context } \\
\hline Mainland China & 12.12 \\
\hline Hong Kong & 45.45 \\
\hline Taiwan & 3.03 \\
\hline US & 15.15 \\
\hline Canada & 12.12 \\
\hline Singapore & 3.03 \\
\hline Multiple locations $(>=2$ ) & 9.09 \\
\hline \multicolumn{2}{|l|}{ Sample size } \\
\hline$<100$ & 30.30 \\
\hline $100+$ & 69.70 \\
\hline \multicolumn{2}{|l|}{ Family SES } \\
\hline Reported & 27.27 \\
\hline No report & 72.73 \\
\hline \multicolumn{2}{|l|}{ Grade level } \\
\hline PreK (including K) & 21.21 \\
\hline Primary & 36.36 \\
\hline Middle & 12.12 \\
\hline Prek \& Primary & 15.15 \\
\hline Primary \& Middle & 15.15 \\
\hline
\end{tabular}

from 3 to 13 years. The majority of the studies (45\%) were conducted in Hong Kong, followed by US (18\%) and Canada (14\%). Figure 2 shows that the overrall mean correlation is moderate, $r=.46,95 \% \mathrm{CI}[.42, .49]$ and significant, $\mathrm{z}(27)=24.50, p<.01$. There was a significant and large variation in correlations between studies, Q $(27)=275.83, p<.01, \mathrm{I}^{2}=90.21$. Table 2 shows that the moderator effect of geographic location is present and students in Hong Kong produced greater correlation between L1 and L2 phonological awareness than those in US or Canada, $\mathrm{Q}(1)=6.60, p<.05$. Moderator analysis on grade level shows L1 and L2 phonological awareness transfer does not differ significantly among participants in pre-kindergarten and primary grades.

\section{Correlation between L1 and L2 vocabulary}

A total of 15 studies comprising 1763 participants examined the relationship between L1 and L2 vocabulary. The age range of study participants varied from 4 to 12:1 years. The majority of the studies were conducted in Hong Kong (33\%), followed by US (25\%) and Canada (17\%). Figure 3 shows the overall correlation was small, $r=0.10,95 \% \mathrm{CI}$ $[.05, .14]$, but significant, $\mathrm{z}(13)=4.30, p<.01$. There was significant and large variation 


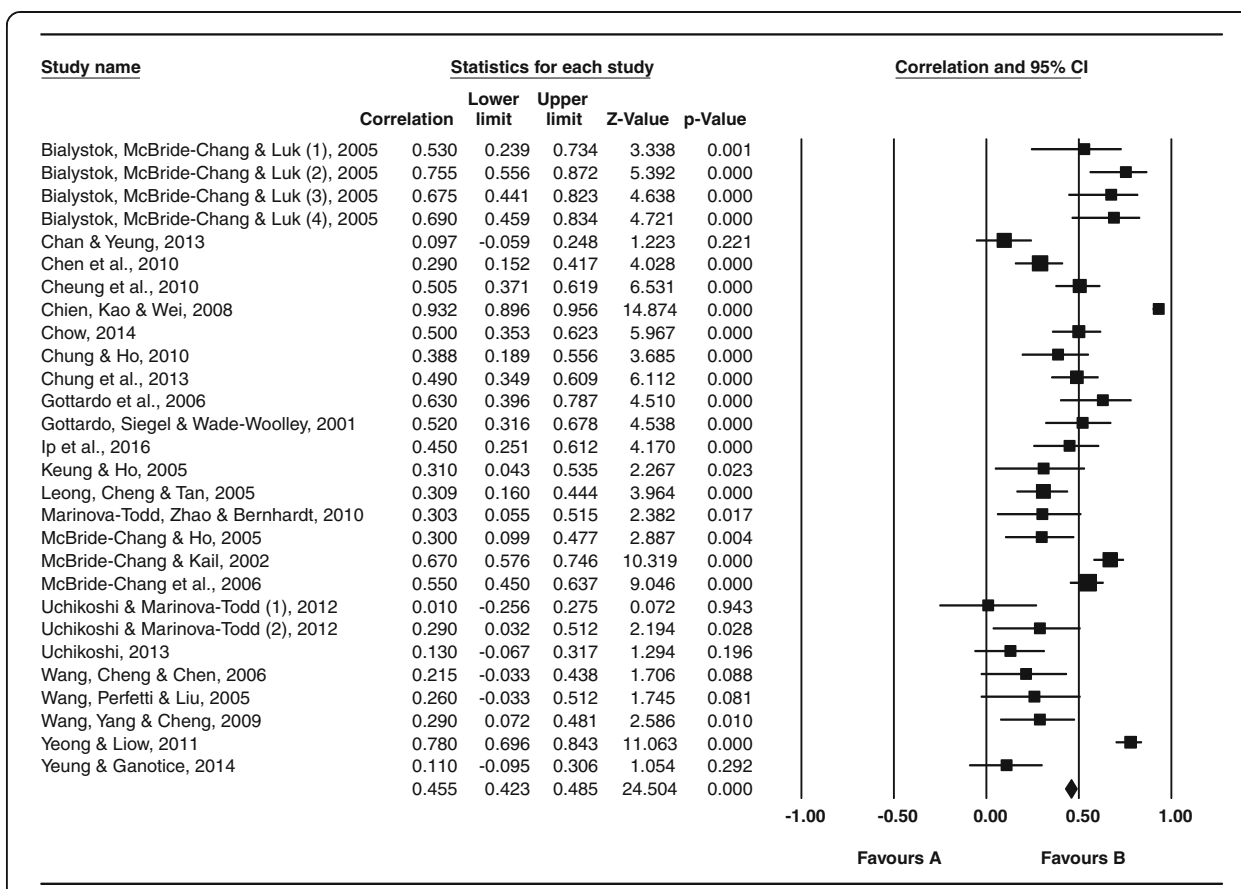

Fig. 2 Overall average correlation and correlation with confidence interval for each study correlating Chinese and English phonological awareness. Note. Overall correlation shows at the bottom of the figure. Diamond shape = overall correlation average across listed studies on graph. Size of square reflects sample size, with bigger square representing a larger sample size (Bialystok, McBride-Chang \& Luk 2005; Chen et al., 2010; Cheung et al., 2010; Chien, Kao \& Wei 2008; Chow 2014; Chung \& Ho 2010; Chung et al., 2013; Gottardo et al., 2006; Gottardo, Siegel \& Wade-Woolley 2001; Ip et al., 2016; Keung \& Ho 2009; Leong et al., 2005; MarinovaTodd et al., 2010; McBride-Chang \& Ho 2005; McBride-Chang \& Kail 2002; McBride-Chang et al., 2006; Uchikoshi \& Marinova-Todd 2012; Uchikoshi 2013; Wang, Cheng \& Chen 2006; Wang, Perfetti \& Liu 2005; Wang, Yang \& Cheng 2009; Yeung \& Chan 2013; Yeong \& Liow 2011; Yeung \& Ganotice 2014)

in correlations across studies, $\mathrm{Q}(13)=63.18, p<.01, \mathrm{I}^{2}=77.84$. Moderator analysis in Table 3 shows that no moderator effect is present as neither of the two Q tests is significant.

\section{Correlation between L1 and L2 decoding skills}

A total of 29 studies comprising 3568 children investigated the relationship between L1 decoding and L2 decoding skills. The age range of the samples varied from 3 to 13 years

Table 2 Number of correlations, correlation, 95\% confidence interval, heterogeneity statistics, difference in $r$ between categories and significance test of categories for moderators of the relationship between Chinese and English phonological awareness

\begin{tabular}{lllllcc}
\hline Moderator variable & $\begin{array}{l}\text { Number of } \\
\text { correlations }\end{array}$ & Correlation & $95 \% \mathrm{Cl}$ & Heterogeneity $\left(\mathrm{I}^{2}\right)$ & $\begin{array}{l}\text { Difference in } r \\
\text { (highest-lowest) }\end{array}$ & $\begin{array}{c}\text { Significance test } \\
\text { of Q test }\end{array}$ \\
\hline $\begin{array}{l}\text { Geographic location } \\
\text { US/CA }\end{array}$ & 12 & $.34^{* *}$ & $.27-.40$ & 65.19 & .21 & $6.60^{*}$ \\
HK & 13 & $.44^{* *}$ & $.40-.48$ & 84.82 & & \\
$\begin{array}{l}\text { Grade levels } \\
\text { PreK }\end{array}$ & 8 & $.42^{* *}$ & $.42-.52$ & 97.34 & .07 & .84 \\
Primary & 9 & $.44^{* *}$ & $.38-.49$ & 140.75 & & \\
\hline
\end{tabular}

HK Hong Kong, SG Singapore, CA Canada

${ }^{*} p<.05$

${ }^{* *} p<.01$ 


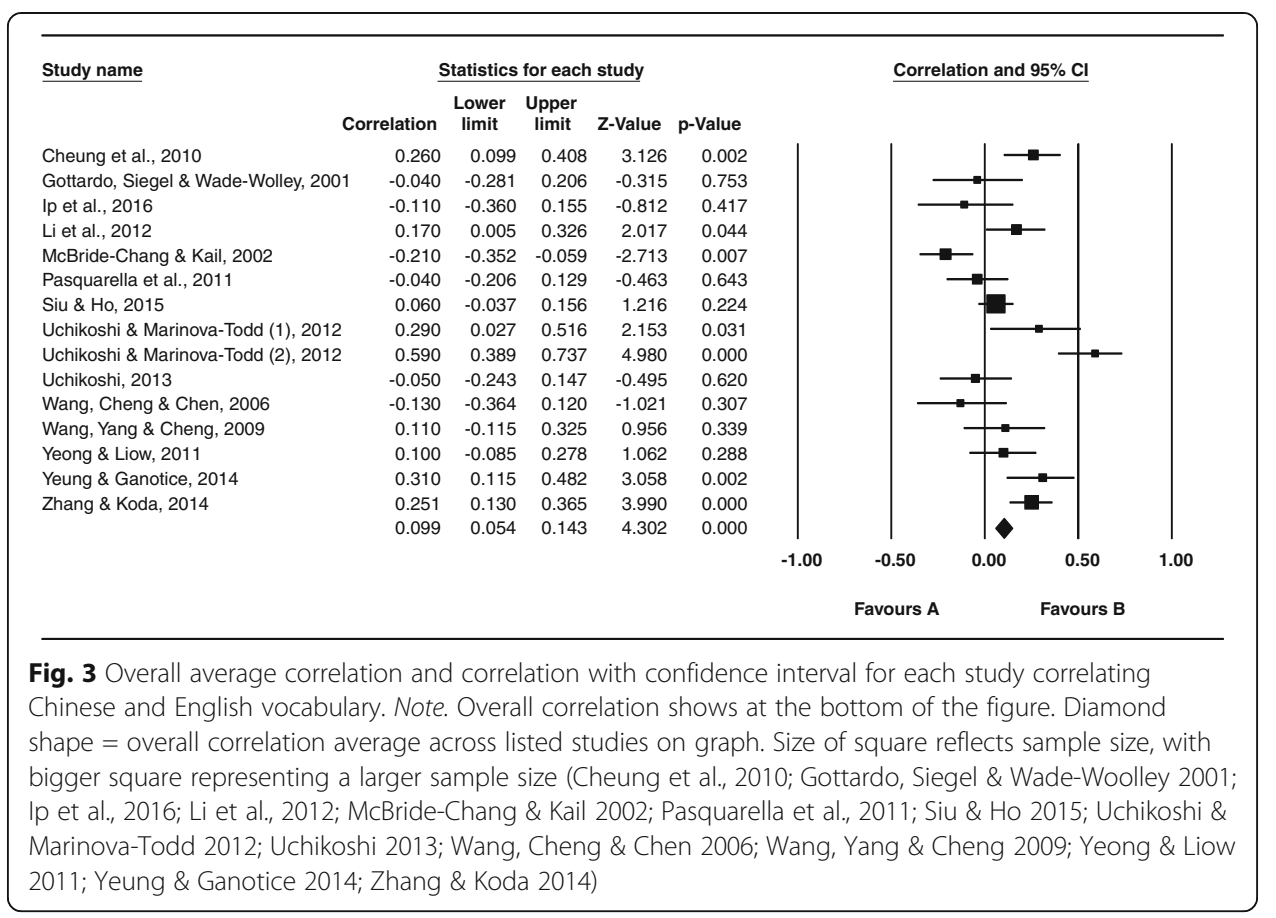

old. Studies were conducted in multiple places such as Hong Kong, US, Canada and Mainland China with the majority of them conducted in Hong Kong (57\%), followed by US (13\%) and Canada (13\%). As Fig. 4 shows, the overrall mean correlation is moderate, $r=.44,95 \% \mathrm{CI}[.41, .47]$, and significant, $\mathrm{z}(28)=26.89, p<.01$. There was a significant and large variation in correlations across studies, $\mathrm{Q}(28)=183.22, p<.01$, $\mathrm{I}^{2}=$ 84.72. Moderator analysis in Table 4 revealed that geographic location, $\mathrm{Q}(1)=86.04, p<.01$, and grade level $\mathrm{Q}(1)=33.50, p<.01$ were all significant moderator variables. Studies conducted in Hong Kong produced greater correlations between L1 and L2 decoding than those conducted in Canada or US. Compared to studies of primary grade students, studies with younger children produced higher correlation between L1 and L2 decoding.

Table 3 Number of correlations, correlation, 95\% confidence interval, heterogeneity statistics, difference in $r$ between categories and significance test of categories for moderators of the relationship between Chinese and English vocabulary

\begin{tabular}{|c|c|c|c|c|c|c|}
\hline Moderator variable & $\begin{array}{l}\text { Number of } \\
\text { correlations }\end{array}$ & Correlation & $95 \% \mathrm{Cl}$ & Heterogeneity $\left(I^{2}\right)$ & $\begin{array}{l}\text { Difference in r } \\
\text { (highest-lowest) }\end{array}$ & $\begin{array}{l}\text { Significance test } \\
\text { of Q test }\end{array}$ \\
\hline \multicolumn{7}{|l|}{ Geographic location } \\
\hline US/CA & 8 & .06 & $-.02-.14$ & 77.19 & .17 & .28 \\
\hline HK & 5 & $.09^{* *}$ & $.02-.15$ & 84.17 & & \\
\hline \multicolumn{7}{|l|}{ Grade levels } \\
\hline PreK & 4 & .20 & $-.03-.18$ & 0 & .14 & 1.12 \\
\hline Primary & 6 & .01 & $-.06-.08$ & .49 & & \\
\hline
\end{tabular}

HK Hong Kong, SG Singapore, CA Canada, CN Mainland China

${ }^{*} p<.05$

** $p<.01$ 


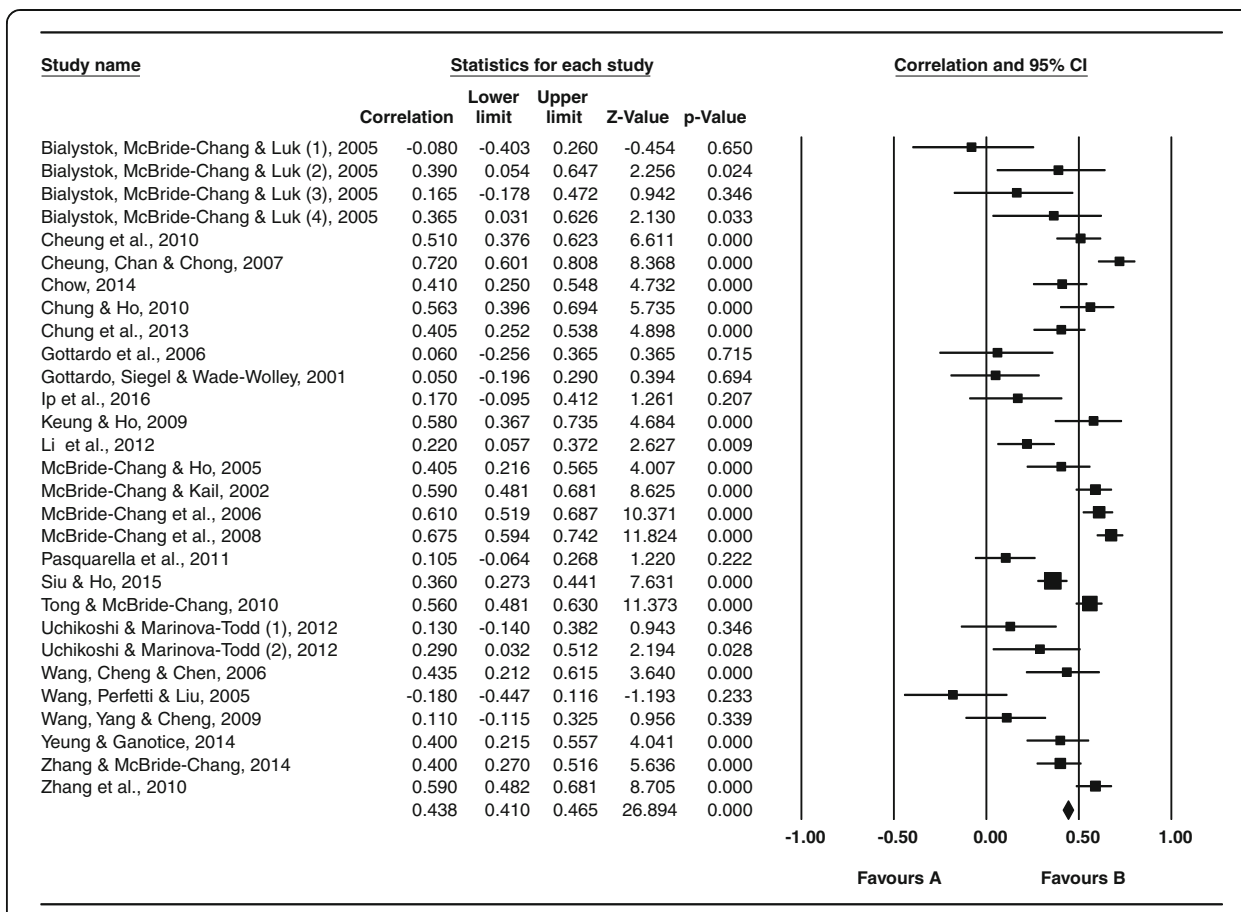

Fig. 4 Overall average correlation and correlation with confidence interval for each study correlating Chinese and English decoding. Note. Overall correlation shows at the bottom of the figure. Diamond shape $=$ overall correlation average across listed studies on graph. Size of square reflects sample size, with bigger square representing a larger sample size (Bialystok, McBride-Chang \& Luk 2005; Cheung et al., 2007; Cheung et al., 2010; Cheung, Chan \& Chong, 2007; Chow 2014; Chung \& Ho 2010; Chung et al., 2013; Gottardo et al., 2006; Gottardo, Siegel \& Wade-Woolley 2001; Ip et al., 2016; Keung \& Ho 2009; Li et al., 2012; McBride-Chang et al., 2006; McBride-Chang \& Kail 2002; McBride-Chang \& Ho 2005; McBride-Chang et al., 2008; Siu \& Ho 2015; Pasquarella et al., 2011; Siu \& Ho 2015; Tong \& McBride-Chang 2010; Uchikoshi \& Marinova-Todd 2012; Wang, Cheng \& Chen 2006; Wang, Perfetti \& Liu 2005; Wang, Yang \& Cheng 2009; Yeung \& Ganotice 2014; Zhang \& McBride-Chang 2014; Zhang et al., 2010)

\section{Correlation between L1 and L2 morphological awareness}

A total of 9 independent correlations investigated the relationship between L1 and L2 morphological awareness among 1070 participants. The age range varied from 5 to 12:1 years and the majority of the studies were conducted in mainland China (38\%) followed by Hong Kong (25\%) and US (25\%). Figure 5 shows that the overall correlation is small, $r=.37,95 \%$ CI $[.31, .42]$, but significant, $\mathrm{z}(8)=12.35, p<.01$. The

Table 4 Number of correlations, correlation, 95\% confidence interval, heterogeneity statistics, difference in $r$ between categories and significance test of categories for moderators of the relationship between Chinese and English decoding

\begin{tabular}{lllllcc}
\hline Moderator variable & $\begin{array}{l}\text { Number of } \\
\text { correlations }\end{array}$ & Correlation & $95 \% \mathrm{Cl}$ & Heterogeneity $\left(\mathrm{I}^{2}\right)$ & $\begin{array}{l}\text { Difference in } r \\
\text { (highest-lowest) }\end{array}$ & $\begin{array}{l}\text { Significance test } \\
\text { of Q test }\end{array}$ \\
\hline $\begin{array}{l}\text { Geographic location } \\
\text { CA/US }\end{array}$ & 11 & $.13^{* *}$ & $.05-.21$ & 32.64 & .48 & $86.04^{* *}$ \\
HK & 17 & $.50^{* *}$ & $.47-.53$ & 78.62 & & \\
$\begin{array}{l}\text { Grade level } \\
\text { PreK }\end{array}$ & 6 & $.56^{* *}$ & $.51-.61$ & 84.17 & .31 & $33.50^{* *}$ \\
Primary & 10 & $.35^{* *}$ & $.30-.40$ & 85.03 & & \\
\hline
\end{tabular}

HK Hong Kong, SG Singapore, CA Canada

${ }^{*} p<.05$

${ }^{* *} p<.01$ 


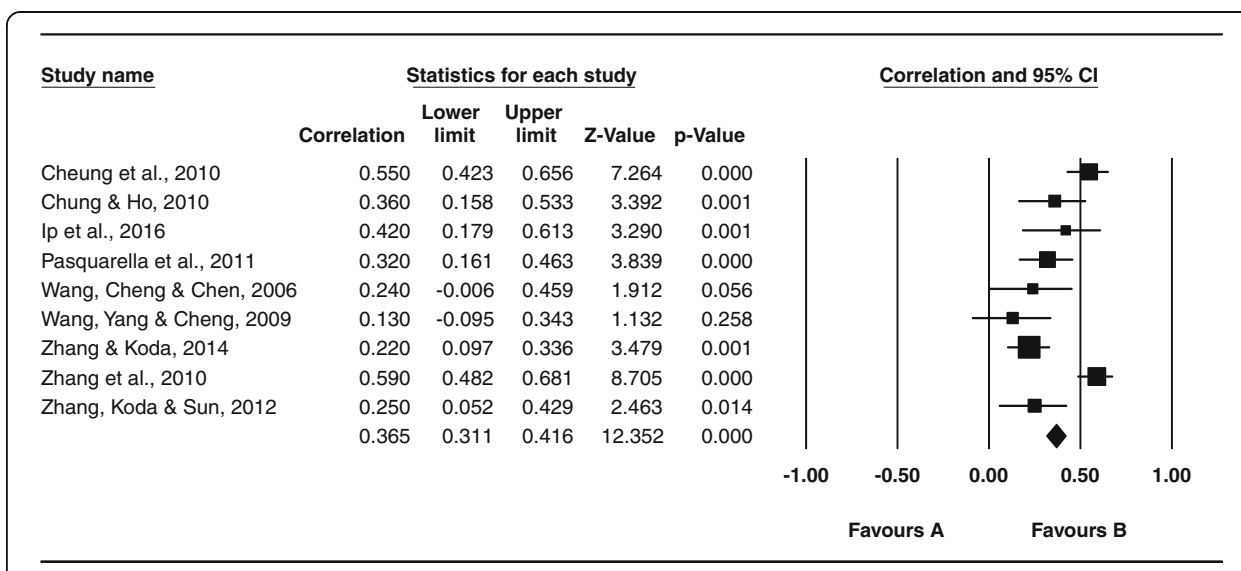

Fig. 5 Overall average correlation and correlation with confidence interval for each study correlating Chinese and English morphological awareness. Note. Overall correlation shows at the bottom of the figure. Diamond shape $=$ overall correlation average across listed studies on graph. Size of square reflects sample size, with bigger square representing a larger sample size (Cheung et al., 2010; Chung \& Ho 2010; Ip et al., 2016; Pasquarella et al., 2011; Wang, Cheng \& Chen 2006; Wang, Yang \& Cheng 2009; Zhang \& Koda 2014; Zhang et al., 2010; Zhang, Koda \& Sun 2012)

variation in correlations between studies was significant Q $(8)=36.15, p<.01$, $\mathrm{I}^{2}=77.87$. Table 5 revealed no moderator effect in terms of L1 and L2 morphological awareness correlation. Studies conducted in China produced greater correlation than those conducted in US or Canada and participants in primary grades produced greater correlation than their peers in middle school, but none of the differences was significant.

\section{Conclusion}

This meta-analysis examined the extent of cross-linguistic transfer between Chinese as the first language (L1) and English as the second language (L2). We found statistically significant correlations between L1 and L2 across four themes including L1 and L2 phonological awareness, L1 and L2 decoding, L1 and L2 vocabulary, and L1 and L2 morphological awareness. The meta-correlations between L1 and L2 vocabulary (.1) and L1 and L2 morphological awareness were rather small (.37), whereas the meta-correlations between L1 and L2 phonological awareness (.46) and L1 and L2 decoding were moderate (.44). There was large

Table 5 Number of correlations, correlation, 95\% confidence interval, heterogeneity statistics, difference in $r$ between categories and significance test of categories for moderators of the relationship between Chinese and English morphological awareness

\begin{tabular}{lcccccc}
\hline Moderator variable & $\begin{array}{l}\text { Number of } \\
\text { correlations }\end{array}$ & Correlation & $95 \% \mathrm{Cl}$ & Heterogeneity $\left(\mathrm{I}^{2}\right)$ & $\begin{array}{l}\text { Difference in } r \\
\text { (highest-lowest) }\end{array}$ & $\begin{array}{c}\text { Significance test } \\
\text { of Q test }\end{array}$ \\
\hline $\begin{array}{l}\text { Geographic location } \\
\text { US/CA }\end{array}$ & 4 & $.28^{* *}$ & $.18-.38$ & 16.69 & .26 & 1.64 \\
CN & 3 & $.36^{* *}$ & $.28-.44$ & 90.90 & & \\
Grade levels & & & & & .26 & 1.64 \\
$\quad \begin{array}{l}\text { Primary } \\
\text { Middle }\end{array}$ & 4 & $.38^{* *}$ & $.18-.38$ & 90.90 & & \\
\hline
\end{tabular}

HK Hong Kong, SG Singapore, CA Canada, CN Mainland China

${ }^{*} p<.05$

** $p<.01$ 
variation across studies in all correlations as indicated by the Q statistics. We also investigated the potential moderator effects of geographic location and grade level and results of these moderator effects vary to a certain extent. Specifically, participants in Hong Kong produced greater cross-linguistic transfer between Chinese and English regarding phonological awareness and decoding skills compared to participants located in U.S. or Canada. Grade level, divided into preschool or kindergarten, primary, and middle school, only significantly moderated the magnitude of correlations between L1 and L2 decoding and younger children produced significantly greater correlation than their older peers.

\section{Cross-linguistic transfer of phonological awareness, decoding, vocabulary and morphological awareness}

Similar to Melby-Lervag and Lervag (2011), who identified a moderate to large correlation ( $r=.60, n=16$ ) between L1 and L2 (English) phonological awareness, we found a reliable moderate correlation between L1 (Chinese) and L2 (English) phonological awareness $(r=.46, n=28)$. Despite the many differences between Chinese and English phonological awareness in terms of sound inventory, syllable structure, and the utilization of suprasegmental features such as tones to mark meaning, there is still significant overlap of skills when learners are required to segment, analyze, manipulate, and assemble speech sounds. These results are in agreement with Cummins' (1979) notions of linguistic interdependence and common underlying proficiency. Even though Chinese and English have highly dissimilar phonological structures, completing the phonological awareness task requires sensitivity to speech sounds and perceptual and attention skills that are common across different phonological systems.

Also similar to Melby-Lervag and Lervag (2011), who identified a small but significant correlation between L1 and L2 vocabulary $(r=.16, n=36)$, we discovered a weak yet significant correlation between L1 and L2 vocabulary $(r=.10, n=15)$. MelbyLervag and Lervag (2011) attributed the smaller correlation to the complex and multifaceted nature of oral language. Note that Melby-Lervag and Lervag (2011) focused on oral language comprehension and oral vocabulary while we focused on oral vocabulary only. Given the lack of cross-language cognates (e.g., words that share similarities in meaning and auditory or visual forms across languages) between Chinese and English, it is not surprising that the correlation in oral vocabulary was small.

Despite the drastic differences between Chinese and English orthography, we found a reliable moderate correlation between L1 and L2 decoding skills $(r=.44, n=29)$. Melby-Lervag and Lervag (2011) noted an overall correlation of .54 $(n=22)$ between L1 and L2 decoding; however, when examined by closeness of writing system, the correlation decreased to $.24(n=4)$ between ideographic (or logographic) orthography and English. How does this cross-language correlation come about? English is an alphabetic language with a deep orthography in that the grapheme-phoneme correspondence in English is quite inconsistent (Georgiou et al., 2008; Ziegler \& Goswami, 2005). To successfully and efficiently decode English words, children need to use a combination of strategies such as grapheme-phoneme correspondence, morphological units, analogy, and sight word/whole-word recognition. Chinese also has a deep orthography and even though the phonetic radicals embedded in some Chinese characters can provide clues to the pronunciation of the character, those cues are quite inconsistent and unreliable (Wang \& Koda, 2005; Shu \& Anderson, 1997; Zhu, 1988). Therefore reading Chinese 
and English both require a combination of phonological awareness, morphological awareness, as well as orthographic processing skills. These common challenges faced by readers of Chinese and English may be the underlying mechanism of the crosslinguistic correlation (Wang \& Koda, 2005).

A unique aspect of the current meta-analysis was the inclusion of meta-correlation calculations for L1 and L2 morphological awareness. All nine studies included in this calculation uniformly examined compound awareness and the overall correlation was small but significant $(r=.37)$. According to the contrastive analysis model (Connor, 1996; Ellis, 1994; Lado, 1957; Odlin, 1989), the structural similarity in terms of how compound words are formed in Chinese and English may be the foundation of crosslinguistic tranfer (Lam \& Sheng, 2016). To be specific, Mandarin and English compound words both use the right-headed structure, wherein the head of the compound is placed on the right whereas the modifier morpheme that specifies the head is on the left (Lam \& Sheng, 2016). For instance, in “教室” [jiao4 shi4], the Chinese word 'classroom', the modifier morpheme on the left - [jiao4] means 'to teach' whereas the head morpheme on the right $-[$ shi4] indicates 'room'.

\section{Moderator effect of geographic location and grade level}

Our review identified two moderators: geographic location and grade level. Geographic location was a significant moderator for two themes. Specifically, studies conducted in Hong Kong produced greater correlations than those conducted in the US and Canada in the areas of phonological awareness and decoding. Consistent with the societal prestige and the economic advantage assoicated with English competence, most children in Hong Kong start learning English at a fairly early age (Kan \& Adamson, 2010). Moreover, under the guidance of Hong Kong government collaborative language policies, which seek to avoid instead of exacerbate tensions between one language versus the other, students are getting greater exposure to English at schools while still maintaining their first language. In other words, the collaborative language policy promotes an additive bilingual environment in Hong Kong, which may have enabled students to maintain their first language while adding a second one and such additive environment is also facilitating the transfer between the two langauges (Lambert, 1975). By contrast, studies of Mandarin-English bilingual children in the United States indicated an early stagnation of these children's Mandarin skills as these children were focusing on learning English and becoming English-dominant (Sheng, 2014). L1, or heritage language attribution has been found in other bilingual groups in the North America context. The subtractive bilingual environement in North America may have led to a suppressed level of crosslinguistic transfer (Cobo-Lewis et al., 2002; Hammer et al., 2008; Sheng, 2014).

Geographic location is a complex variable that entails cultural, educational, socialpolitical, and linguistic differences. Our meta-analysis is meant to provide a comprehensive and fair view of the current cumulative evidence on cross-linguistic transfer between Chinese and English and the evidence we were able to gather between the two languages is situated in the complex sociocultural, political and educational environment where both langauges are currently being used. We attempted to investigate a number of variables such as current use of English/Chinese, age of onset of English learning, language dominance, and language of instruction at school, which may help to disaggregate the multiplex geographic location factor. However, most studies did not 
provide detailed information on these variables, making it impossible to quantify the potential influences of these important factors. To further our understanding of how L1 and L2 crosslinguistic transfer takes place, future studies should more carefully document the linguistic and educational background and language usage patterns of their participants.

Grade level also moderated the relationship between L1 and L2 decoding. Studies with Pre-K children produced a higher overall correlation $(r=.56)$ than those with primary grade childre $(r=.35)$. Grade level could be seen as a proxy for duration of L2 learning and by extension, L2 proficiency. This finding was consistent with the prediction of the Revised Hierarchical Model (RHM), which proposed that younger bilinguals, or those at the beginning stage of L2 acquisition, are more inclined to rely on the connection between L1 and L2 translation equivalents to access the meaning of L2 words (Kroll \& Stewart, 1994). As children develop their L2 skills, they are more able to directly access the meanings of L2 words, hence the use of L1 words as a mediation will gradually diminish (Sheng et al., 2013), thereby the cross-language correlation also decreases.

\section{Limitations and future directions}

One major limitation of our meta-analysis is the small sample size. We included 33 articles that were published in peer-reviewed English journals since 2000. We included peer-reviewed articles only because the evidence has undergone rigorous scrutiny. We focused on English journals because the original articles are more widely accessible to the broader scientific community. The meta-analysis conducted by Melby-Lervag and Lervag (2011) included a total of 47 studies with a variety of L1 s represented. Our study focused exclusively on English and Chinese. The majority of studies included in our review focused on code-related skills such as phonological awareness and decoding. Therefore, more research is needed in the areas of morphological awareness and vocabulary. Second, we were unable to examine the impact of potential moderators such as family SES, immigration status, and language dominance due to the lack of a sufficient number of studies. Future studies need to more thoroughly report participants' demographic and linguistic background to enable detailed analyses of the contribution of these factors. Third, we share a common concern as noted by Melby-Lervag and Lervag (2011) that the use of psychometrically equated measurement in L1 and L2 is needed to allow fair and valid comparisons between L1 and L2 skills (e.g., Alderson, 1984; Cummins, 1991). Finally, as customary in studies of cross-linguistic transfer (e.g., Proctor et al., 2006; Swanson et al., 2008) and similar to Melby-Lervag and Lervag's meta-analysis, we relied on correlational evidence to draw conclusions regarding potential crosslinguistic transfer. Nevertheless, correlation does not imply causation. Therefore, we discourage the interpretation of the evidence as causal effect from L1 to L2. Instead, we can only say that the two languages share common variances in the linguistic skills examined and there is potential for bi-directional, L1-to-L2, and L2-to-L1 influences. Future studies should utilize designs that are more amenable to examining the causal relationship between L1 and L2 skill development.To conclude, this metaanalysis identified significant cross-linguistic transfer between Chinese and English in the area of phonological awareness, decoding, vocabulary and morphological awareness. These results are strikingly similar to previous analyses that primarily focused on IndoEuropean languages (Melby-Lervag \& Lervag, 2011). These findings suggest that 
linguistic distance between a bilingual's two languages does not necessarily impede the growth of the two languages and the positive transfer of skills from one to the other. This proposal is consistent with the view that vocabulary and literacy in the bilingual's two languages are subserved by the same universal common underlying proficiency.

\section{Acknowledgements}

The authors thank Dakota Schiel and Marysabel Guillen for their assistance of data collection.

Funding

This study was supported by a research grant from college of education in the university of Texas at Austin to the first author.

\section{Authors' contributions}

Conceptualization: MY LS. Data curation: MY. Formal analysis: MY LS NC. Funding acquisition: MY. Writing - original draft: MY LS. Writing - review \& editing: MY LS. All authors read and approved the final manuscript.

Ethics approval and consent to participate

Not applicable.

\section{Competing interests}

The authors declare that they have no competing interests

\section{Publisher's Note}

Springer Nature remains neutral with regard to jurisdictional claims in published maps and institutional affiliations.

Received: 19 June 2017 Accepted: 6 September 2017

Published online: 02 October 2017

\section{References}

(Studies marked with * are included in the meta-analysis)

Abbott, M. L. (2006). ESL reading strategies: Differences in Arabic and Mandarin speaker test performance. Language learning, 56(4), 633-670.

Adams, M. J. (1990). Beginning to read: Thinking and learning about print. Cambridge, MA: MIT Press.

Alderson, J. C. (1984). Reading in a foreign language: A reading or a language problem? In C. Alderson \& A. H. Urquhart (Eds.), Reading in a foreign language (pp. 1-24). London: Longman.

Altman, D. G. (1990). Practical statistics for medical research. CRC press.

Bax, L., Yu, L. M., Ikeda, N., \& Moons, K. G. (2007). A systematic comparison of software dedicated to meta-analysis of causal studies. BMC Medical Resarch Methodlogy, 7(1), 40.

Bialystok, E., McBride-Chang, C., \& Luk, G. (2005). Bilingualism, language proficiency, and learning to read in two writing systems. Journal of Educational Psychology, 97(4), 580-590.

Borenstein, M., Hedges, L. V., Higgins, J. P. T., \& Rothestein, H. R. (2009). Introduction to meta-analysis. West Sussex, UK: John Wiley \& Sons.

Carlisle, J. F. (2000). Awareness of the structure and meaning of morphologically complex words: Impact on reading. Reading and writing, 12(3), 169-190.

Casalis, S., \& Louis-Alexandre, M. F. (2000). Morphological analysis, phonological analysis and learning to read French: A longitudinal study. Reading and Writing: An Interdisciplinary Journal, 12, 303-335.

Chen, H. C., d'Arcais, G. B. F., \& Cheung, S. L. (1995). Orthographic and phonological activation in recognizing Chinese characters. Psychological Research, 58(2), 144-153.

${ }^{*}$ Chen, X., Xu, F., Nguyen, T. K., Hong, G., \& Wang, Y. (2010). Effects of cross-language transfer on first-language phonological awareness and litearcy skills in Chinese children receiving English instruction. Journal of Educational Psychology, 102(3), 712-728.

Cheng, C.C. (1991). Quantifying affinity among Chinese dialects. Journal of Chinese Linguistic Monograph Series, (3), 76-110

${ }^{*}$ Cheung, H., Chan, M., \& Chong, K. (2007). Use of orghographic knowledge in reading by Chinese-English bi-scriptal children. Language Learning, 57(3), 469-505.

${ }^{*}$ Cheung, H., Chung, K. K. H., Wong, S. W. L., Mc-Bride-Chang, C., Penney, T. B., \& Ho, C. S. H. (2010). Speech perception, metalinguistic awareness, reading, and vocabulary in Chinese-English bilingual children. Journal of Educational Psychology, 102(2), 367-380.

*Chien, C. N., Kao, L. H., \& Wei, L. (2008). The role of phonological awareness development in young Chinese EFL learners. Language Awareness, 17(4), 271-288.

Choi, P. (2003). 'The best students will learn English': Ultra-utilitarianism and linguistic imperialism in education in post1997 Hong Kong. Journal of Education Policy, 18, 673-694.

${ }^{*}$ Chow, B. W. Y. (2014). The differential roles of paired associate learning in Chinese and English word reading abilities in biligual children. Reading and Writing, 27, 1657-1672.

Chow, B. W. Y., McBride-Chang, C., \& Burgess, S. (2005). Phonological processing skills and early reading abilities in Hong Kong Chinese kindergarteners learning to read English as a second language. Journal of Educational Psychology, 97(1), 81. 
${ }^{*}$ Chung, K. K. H., \& Ho, C. S. H. (2010). Second language learning difficulties in Chinese children with dyslexia: What are the reading-related cognitive skills that contribute to English and Chinese word reading? Journal of Learning Disabilities, 43(3), 195-211.

${ }^{*}$ Chung, K. K. H., McBride-Chang, C., Cheung, H., \& Wong, S. W. L. (2013). General autitory processing, speech perception and phonological awarenaess skills in Chinese-English biliteracy. Journal of Research in Reading, 36(2), 202-222.

Cisero, C. A., \& Royer, J. (1995). The development and cross-language transfer of phonological awareness. Contemporary Educational Psychology, 20, 275-303.

Cobo-Lewis, A. B., Pearson, B. Z., Eilers, R. E., \& Umbel, V. C. (2002). Effects of bilingualism and bilingual educaion on oral and written English skills: A multifactor study of standardized test outcomes. In D. K. Oller \& R. E. Eilers (Eds.), Language and literacy in bilingual children (pp. 64-97). Buffalo, NY: Multilingual Matters.

Comeau, L., Cormier, P., Grandmaison, E., \& Lacroix, D. (1999). A longitudinal study of phonological processing skills in children learning to read in a second language. Journal of Educational Psychology, 91, 29-43.

Connor, U. (1996). Contrastive rhetoric: Cross-cultural aspects of second-language writing. Cambridge: Cambridge University Press.

Cummins, J. (1979). Linguistic interdependence and the educational development of bilingual children. Review of Educational Research, 49, 222-251.

Cummins, J. (1991). Interdependence of first-and second-language proficiency in bilingual children.Language processing in bilingual children, 70-89.

D'Angiulli, A., Siegel, L. S., \& Serra, E. (2001). The development of reading in Italian and English in bilingual children. Applied PsychoLinguistics, 22, 479-507.

Deacon, S. H., \& Kirby, J. R. (2004). Morphological awareness: Just more phonological awareness? The roles of morphological and phonological awareness in reading development. Applied PsychoLinguistics, 25, 223-238.

Dunn, L. M., \& Dunn, L. M. (1997). Peabody picture vocabulary test (3rd ed.). Circle Pines, MN: American Guidance Service.

Durgunoğlu, A. Y. (2002). Cross-linguistic transfer in literacy development and implications for language learners. Annals of Dyslexia, 52, 189-204.

Durgunoglu, A. Y., Navy, W. W., \& Hancin-Bhatt, B. J. (1993). Cross-language transfer of phonemic awareness. Journal of Educational Psychology, 85, 453-465.

Ellis, R. (1994). The study of second language acquisition. Oxford University.

Georgiou, G. K., Parrila, R., \& Papadopoulos, T. C. (2008). Predictors of word decoding and reading fluency across language varying in orthographic consistency. Journal of Educational Psychology, 100(3), 566.

*Gottardo, A., Chiappe, P., Yan, B., Siegel, L., \& Gu, Y. (2006). Relationships between first and second language phonological processing skills and reading in chinese-English speakers living in English-speaking contexts. Educational Psychology, 26(3), 367-393.

*Gottardo, A., Siegel, L. S., \& Wade-Woolley, L. (2001). Factors related to English reading performance in children with Chinese as a first language: More evidence of cross-language transfer. Journal of Educational Psychology 93(3), 530-542.

Hammer, C. S., Lawrence, F. R., \& Miccio, A. W. (2008). Exposure to English before and after entry to head start: Bilingual children's receptive language growth in Spanish and English. Int J Biling Educ Biling, 11, 30-56.

Hashimoto, O. Y. (1972). Phonology of Cantonese. England: Cambridge University Press.

Hasselblad, V., \& Hedges, L. V. (1995). Meta-analysis of screening and diagnostic tests. Psychological Bulletin, 117, 167-178.

Hedges, L. V., \& Olkin, I. (1985). Statistical methods for meta-analysis. Orlando, FL: Academic Press.

Hua, Z. (2002). Phonological development in specific contexts: Studies of Chinese-speaking children (Vol. Vol.3) Multilingual matters.

Huang, H. S., \& Hanley, J. R. (1995). Phonological awareness and visual skills in learning to read Chinese and English. Cognition, 54(1), 73-98.

*Ip, K. I., Hsu, L. S. J., Arredondo, M. M., Tardif, T., \& Kovelman, I. (2016). Brain bases of morphological processing in Chinese-English bilingual children. Developmental Science.

Kan, V., \& Adamson, B. (2010). Language policies for Hong Kong schools since 1997. London Review of Education, 8(2), $167-176$.

Keenan, J. M., Betjemann, R. S., \& Olson, R. K. (2008). Reading comprehension tests vary in the skills they assess: Differential dependence on decoding and oral comprehension. Scientific Studies of Reading, 12(3), 281-300.

*Keung, Y. C., \& Ho, C. S. H. (2009). Transfer of reading-related cognitive skills in learning to read Chinese (L1) and English (L2) among Chinese elementary school children. Contemporary Educational Psychology, 34, 103-112.

Kim, J. S., \& Quinn, D. M. (2013). The effects of summer reading on low-income children's literacy achievement from kindergarten to grade 8: A meta-analysis of classroom and home interventions. Review of Educational Research, 83(3), 386-431.

Kroll, J. F., \& Stewart, E. (1994). Category interference in translation and picture naming: Evidence for asymmetric connections between bilingual memory representations. Journal of Memory and Language, 33, 149-174.

Kroll, J., Van Hell, J. G., Tokowicz, N., \& Green, D. W. (2010). The revised hierachical model: A critical review and assessment. Bilingualism: Language and Cognition, 13, 373-381.

Lado, R. (1957). Linguistics across cultures: Applied linguistics for language teachers. Ann Arbor, Ml: University of Michigan Press.

Lam, B. P. W., \& Sheng, L. (2016). The Development of Morphological Awareness in Young Bilinguals: Effects of Age and L1 Background. Journal of Speech, Language, and Hearing Research, 59(4), 732-744.

Lambert, W. E. (1975). Culture and language as factors in learning and education. In A. Wolfgang (Ed.), Education of immigrant students. Toronto: Ontario Institute for Studies in Education.

*Leong, C. K., Cheng, P. W., \& Tan, L. H. (2005). The role of sensitivity to rhymes, phonemes and tones in reading English and Chinese pseudowords. Reading and Writing, 18, 1-26.

*Li, T., McBride-Chang, C., Wong, A., \& Shu, H. (2012). Longitudinal predictors of spelling and reading comprehension in Chinese as an L1 and English as an L2 in Hong Kong Chinese children. Journal of Educational Psychology, 104(2), $286-301$.

Lipsey, M. W., \& Wilson, D. B. (1993). The efficacy of psychological, educational, and behavioral treatment: Confirmation from meta-analysis. American Psychologist, 48, 1181-1209. 
*Luo, Y. C., Chen, X., \& Geva, E. (2014). Concurrent and longitudinal cross-linguistic transfer of phonological awareness and morphological awareness in Chinese-English bilingual children. Written Language \& Literacy, 17(1), 89-115.

*Marinova-Todd, S. H., Zhao, J., \& Bernhardt, M. (2010). Phonological awareness skills in the two languages of mandarinEnglish bilingual children. Clinical Linguistics \& Phonetics, 24(4-5), 387-400.

*McBride-Chang, C., Cheung, H., Chow, B. W. Y., Chow, C. S. L., \& Choi, L. (2006). Metalinguistic skills and vocabulary knowledge in Chinese (L1) and English (L2). Reading and Writing, 19, 695-716.

*McBride-Chang, C., \& Ho, C. S. H. (2005). Predictors of beginning reading in Chinese and English: A 2-year longitudinal study of Chinese kindergarteners. Scientific Studies of Reading, 9(2), 117-144.

*McBride-Chang, C., \& Kail, R. V. (2002). Cross-cultural similarities in the predictors of reading acquisition. Child Development, 73(5), 1392-1407.

*McBride-Chang, C., Tong, X., Shu, H., Wong, A. M. Y., Leung, K. W., \& Tardif, T. (2008). Syllable, phoneme, and tone: Psycholinguistic units in early Chinese and English word recognition. Scientific Studies of Reading, 12(2), 171-194.

Melby-Lervag, M., \& Lervag, A. (2011). Cross-linguistic transfer of oral language, decoding, phonological awareness and reading comprehension: A meta-analysis of the correlational evidence. Journal of Research in Reading, 34(1), 114-135.

Metsala, J. L. (1999). Young children's phonological awareness and nonword repetition as a function of vocabulary development. Journal of Educational Psychology, 91(1), 3.

Odlin, T. (1989). Language transfer: Cross-linguistic influence in language learning. Cambridge University Press.

Pakir, A. (2004). Medium-of-instruction policy in Singapore. Medium of instruction policies: Which agenda, 117-133.

*Pasquarella, A., Chen, X., Lam, K., \& Luo, Y. C. (2011). Cross-language transfer of morpological awareness in ChineseEnglish bilinguals. Journal of Researach in Reading, 34(1), 23-42.

Proctor, C. P., Carlo, M., August, D., \& Snow, C. (2006). The intriguing role of Spanish language vocabulary knowledge in predicting English reading comprehension. Journal of Educational Psychology, 98(1), 159-169. https://doi.org/10.1037/0022-0663.98.1.159.

Riches, C., \& Genesee, K. (2006). Literacy: Crosslinguistic and crossmodal issues. In D. Genesee, F. Lindholm-Leary, K. Saunders, \& W. M. Christian (Eds.), Educating English language learners: A synthesis of research evidence (pp. 64-108). New York, NY: Cambridge University press.

Shanahan, T., MacArthur, C. A., Graham, S., \& Fitzgerald, J. (2006). Relations among oral language, reading, and writing development. Handbook of writing research, 171-183.

Sheng, L. (2014). Lexical-semantic skills in bilingual children who are becoming English-dominant: A longitudinal study. Bilingualism: Language and Cognition, 17, 556-571.

Sheng, L., Bedore, L. M., Pena, E. D., \& Fiestas, C. (2012). Semantic development in Spanish-English bilingual children: Effects of age and language experience. Child Development, 84(3), 1034-1045.

Sheng, L., Bedore, L. M., Peña, E. D., \& Fiestas, C. E. (2013). Semantic development in Spanish-English bilingual children: Effects of age and language experience. Child Development, 84, 1034-1045.

Shu, H., \& Anderson, R. C. (1997). Role of radical awareness in the character and word acquisition of Chinese children. Reading Research Quarterly, 32(1), 78-89.

Shu, H., McBride-Chang, C., Wu, S., \& Liu, H. (2006). Understanding Chinese development dyslexia: Morphological awareness as a core cognitive construct. Journal of Educational Psychology, 98(1), 122.

*Siu, C. T. S., \& Ho, C. S. H. (2015). Cross-language transfer of syntactic skills and reading comprehension among young Cantonese-English bilingual students. Reading Research Quarterly, 50(3), 313-336.

Snow, C., Burns, S., \& Griffin, P. (1998). Preventing reading difficulties in young children. Washington, DC: National Academy Press.

Sun, C. (2006). Chinese: A linguistic introduction. Cambridge University Press.

Swanson, H. L., Rosston, K., Gerber, M., \& Solari, E. (2008). Influence of oral language and phonological processing on children's bilingual reading. Journal of School Psychology, 46, 413-429. https://doi.org/10.1016/j.jsp.2007.07.002

*Tong, X., \& McBride-Chang, C. (2010). Chinese-English biscriptal reading: Cognitive component skills across orthographies. Reading and Writing, 23, 293-310.

Tung, P., Lam, R., \& Tsang, W. K. (1997). English as a medium of instruction in post-1997 Hong Kong: What students, teachers, and parents think. Journal of Pragmatics, 28, 441-459.

*Uchikoshi, Y. (2013). Predictors of English reading comprehension: Cantonese-speaking English language learners in the U.S. Reading and Writing, 26, 913-939.

*Uchikoshi, Y., \& Marinova-Todd, S. H. (2012). Language proficiency and early literacy skills of Cantonese-speaking English language learners in the U.S. and Canada. Reading and Writing, 25, 2107-2129.

U.S. Census Bureau. (2013). Nearly 3 Million U.S. Residents Speak Chinese at Home. Retrived from https://www.census.gov/ newsroom/press-releases/2013/cb13-r90.html

Verhoeven, L. T. (1994). Transfer in bilingual development: The linguistic interdependence hypothesis revisited. Language Learning, 44(3), 381-415.

Wagner, R. K., \& Torgesen, J. K. (1987). The nature of phonological processing and its causal role in the acquisition of reading skills. Psychological Bulletin, 101, 192-212.

Wang, M., Cheng, C., \& Chen, S. W. (2006). Contribution of morphological awareness to Chinese-English biliteracy acquisition. Journal of Educational Psychology, 98(3), 542.

Wang, M., \& Koda, K. (2005). Commonalities and differences in word identification skills among learners of English as a second language. Language Learning, 55(1), 71-98.

Wang, M., Koda, K., \& Perfetti, C. A. (2003). Alphabetic and nonalphabetic L1 effects in English word identification: A comparison of Korean and Chinese English L2 learners. Cognition, 87(2), 129-149.

*Wang, M., Perfetti, C. A., \& Liu, Y. (2005). Chinese-English biliteracy acquisition: Cross-language and writing system transfer. Cognition, 97, 67-88.

*Wang, M., Yang, C., \& Cheng, C. (2009). The contributions of phonology, orthography, and morphology in ChineseEnglish biliteracy acquisition. Applied PsychoLinguistics, 30, 291-314.

*Yeong, S. H. M., \& Liow, S. J. R. (2011). Cognitive-linguistic foundations of early spelling development in bilinguals. Journal of Educational Psychology., 103(2), 470-488. 
*Yeung, S. S., \& Chan, C. K. K. (2013). Phonological awareness and oral language proficiency in learning to read English among Chinese kindergarten children in Hong Kong. British Journal of Educational Psychology, 83, 550-568.

*Yeung, S. S., \& Ganotice, F. A. (2014). The role of phonological awareness in bilinteracy acquisition among Hong Kong Chinese kindergarteners who learn English as a second language (ESL). Asia-Pacific Edu Res, 23(3), 333-343.

*Zhang, J., Anderson, R. C., Li, H., Dong, Q., Wu, X., \& Zhang, Y. (2010). Cross-language transfer of insight into the structure of compound words. Reading and Writing, 23, 311-336.

*Zhang, D., \& Koda, K. (2014). Awareness of derivation and compounding in Chinese-English biliteracy acquistion. International Journal of Bilingual Education and Bilingualism, 17(1), 55-73.

*Zhang, D., Koda, K., \& Sun, X. (2012). Morphological awareness in biliteracy acquisition: A study of young Chinese EFL readers. International Journal of Bilingualism., $0(0), 1-16$.

*Zhang, J., \& McBride-Chang, C. (2014). Auditory sensitivity, speech perception, L1 Chinese, and L2 English reading abilities in Hong Kong Chinese children. Developmental Psychology, 50(4), 1001-1013.

Zhou, X., \& Marslen-Wilson, W. (1996). Direct visual access is the only way to access the Chinese mental lexicon. In Proceedings of the 18th annual conference of the Cognitive Science Society (pp. 714-719). Erlbaum Hillsdale, NJ.

Zhu, X. (1988). Analysis of the cuing function of the phonetic in modern Chinese. In Proceedings of the symposium on Chinese language and character (pp. 85-104). Beijing, China: Guang Ming Daily Press.

Ziegler, J. C., \& Goswami, U. (2005). Reading acquisition, developmental dyslexia, and skilled reading across languages: a psycholinguistic grain size theory. Psychological bulletin, 131(1), 3.

\section{Submit your manuscript to a SpringerOpen ${ }^{\circ}$ journal and benefit from:}

- Convenient online submission

- Rigorous peer review

- Open access: articles freely available online

- High visibility within the field

- Retaining the copyright to your article

Submit your next manuscript at $>$ springeropen.com 